\title{
Preferential Solvation of Acetaminophen in Propylene Glycol + Water Co-Solvent Mixtures
}

\author{
María Ángeles Peña ${ }^{1}$, Daniel Ricardo Delgado² and Fleming Martínez ${ }^{2, *}$ \\ ${ }^{1}$ Departamento de Ciencias Biomédicas, Facultad de Farmacia, Universidad de Alcalá, Alcalá de Henares, \\ Madrid, Spain \\ ${ }^{2}$ Grupo de Investigaciones Farmacéutico Fisicoquímicas, Departamento de Farmacia, Facultad de Ciencias, \\ Universidad Nacional de Colombia, A.A. 14490, Bogotá D.C., Colombia
}

\begin{abstract}
The preferential solvation parameters defined as the differences between the local mole fraction of solvents around analgesic drug acetaminophen and those for the bulk co-solvent composition in propylene glycol + water mixtures were derived from their thermodynamic properties by means of the inverse Kirkwood-Buff integrals (IKBI) and the quasi-lattice-quasi-chemical (QLQC) methods. It is found that acetaminophen is sensitive to solvation effects, so according to IKBI method the preferential solvation parameter $\delta x_{\mathrm{PG}, \mathrm{A}}$, is negative in water-rich mixtures but positive in medium compositions and in co-solvent-rich mixtures. It is conjecturable that in water-rich mixtures the hydrophobic hydration around the aromatic ring and methyl group present in the drug plays a relevant role in the solvation. The bigger drug solvation by co-solvent in mixtures of similar solvent proportions and in propylene glycol-rich mixtures could be due mainly to polarity effects. Otherwise, according to QLQC method, this drug is preferentially solvated by the co-solvent in all the mixtures.
\end{abstract}

Keywords: Acetaminophen, propylene glycol, solubility, inverse Kirkwood-Buff integrals, preferential solvation.

\section{INTRODUCTION}

Acetaminophen (N-(4-hydroxyphenyl)ethenamide, CAS RN [103-90-2], Figure 1, A or ACP) is also known as paracetamol and is a drug commonly used in current therapeutics because of its analgesic and antipyretic effects. This analgesic drug is specially indicated in the treatment of several minor diseases presented mainly by pediatric patients [1, 2].

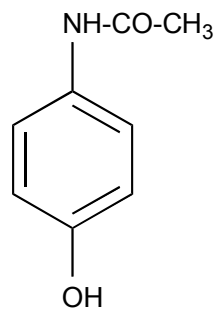

Figure 1: Molecular structure of acetaminophen.

It is well known that the solubility of drugs in cosolvent mixtures knowledge is very important for pharmaceutical and chemical scientists involved in several development stages such as drug purification and design of liquid medicines [3]. Although cosolvency, as good solubilizing technique has been employed in pharmacy for centuries, it is just recently that the mechanisms involved to increase or decrease drugs solubility have been approached from a rigorous

${ }^{*}$ Address correspondence to this author at the Grupo de Investigaciones Farmacéutico Fisicoquímicas, Departamento de Farmacia, Facultad de Ciencias, Universidad Nacional de Colombia, A.A. 14490, Bogotá D.C., Colombia; Tel: 571-3165000-14608; Fax: 571-3165060;

E-mail: fmartinezr@unal.edu.co physicochemical point of view [4]. In this way, some thermodynamic researches about solubility of ACP in aqueous co-solvent mixtures were published based on the enthalpic and entropic contributions to the respective Gibbs energies of solution, mixing and solvation [2, 5]. Nevertheless, the drug preferential solvation, i.e. the co-solvent specific composition around the drug molecules has not been completely studied for this analgesic drug. Therefore, the main goal of this paper is to evaluate the preferential solvation of ACP in propylene glycol + water co-solvent mixtures, based exclusively on thermodynamic definitions. As it is well known, propylene glycol and ethanol are the more widely used co-solvents in the design of liquid pharmaceutical dosage forms [4]. Even more, several products using aqueous mixtures of propylene glycol as vehicle have been described in the literature [4]. Thus, this work is a continuation of the one presented previously in the literature about the behavior of this drug in some ethanol + water mixtures [6].

The inverse Kirkwood-Buff integrals (IKBI) are a powerful tool for evaluating the preferential solvation of non-electrolytes in co-solvent mixtures, describing the local compositions around the solute with respect to the different components present in the solvent mixture [710]. Applied to our research now, this treatment depends on the values of the standard molar Gibbs energies of transfer of ACP from neat water to the propylene glycol + water co-solvent mixtures and the excess molar Gibbs energy of mixing for the co-solvent 
binary mixtures free of drug. In similar way, quasilattice quasi-chemical (QLQC) approach is also useful to do evaluate preferential solvation although is not too much exact as IKBI approach is. This method supposes that the number of nearest neighbors a molecule has (the lattice parameter $Z$ ) is the weighted mean of the lattice parameter of the pure components. It also presumes that the interaction energy of a molecule of any component with others is independent of the nature of the neighbors. The model also assumes that ideal volumes and entropies of mixing take place. The main advantage of this method is that non-derivative functions are required as they are in the case of the IKBI method [7]. Therefore, in this paper the IKBI and QLQC approaches are applied to evaluate the preferential solvation of ACP in the binary mixtures conformed by propylene glycol (PG) and water (W). The results are expressed in terms of the preferential solvation parameter $\left(\delta x_{\mathrm{PG}, \mathrm{A}}\right)$ of the solute by the cosolvent propylene glycol according to the mixtures composition.

\section{THEORETICAL CALCULATIONS}

\section{BACKGROUND}

AND

The Kirkwood-Buff integrals $\left(G_{i, \mathrm{~A}}\right)$ are given by the following expression:

$$
G_{i, \mathrm{~A}}=\int_{0}^{r_{\mathrm{cor}}}\left(g_{i, \mathrm{~A}}-1\right) 4 \pi r^{2} d r
$$

Here $g_{i, A}$ is the pair correlation function for the molecules of the solvent $i$ in the PG + water mixtures around the solute ACP (indicated as $A$ in the equations), $r$ the distance between the centers of the molecules of ACP and PG or water and $r_{\text {cor }}$ is a correlation distance for which $g_{i, \mathrm{~A}}\left(r>r_{\text {cor }}\right) \approx 1$. Thus, for all distances $r>r_{\text {cor }}$ up to infinite, the value of the integral is essentially zero. Therefore, the results are expressed in terms of the preferential solvation parameter $\delta x_{i, \mathrm{~A}}$ for the solute in solution by the component solvents $P G$ and water [11, 12]. For PG this parameter is defined as:

$\delta x_{\mathrm{PG}, \mathrm{A}}=x_{\mathrm{PG}, \mathrm{A}}^{L}-x_{\mathrm{PG}}=-\delta x_{\mathrm{W}, \mathrm{A}}$

Where $X_{P G}$ is the mole fraction of $P G$ in the bulk solvent mixture and $x_{\mathrm{PG}, \mathrm{A}}^{L}$ is the local mole fraction of $\mathrm{PG}$ in the environment near to the drug. If $\delta x_{\mathrm{PG}, \mathrm{A}}>0$ then the solute ACP is preferentially solvated by PG; on the contrary, if it is $<0$ the drug is preferentially solvated by water, within the correlation volume,
$V_{\text {cor }}=(4 \pi / 3) r_{\text {cor }}^{3}$, and the bulk mole fraction of PG, $x_{\mathrm{PG}}$. Values of $\delta x_{\mathrm{PG}, \mathrm{A}}$ are obtainable from those of $\mathrm{G}_{\mathrm{PG}, \mathrm{A}}$, and these in turn, from thermodynamic data of the cosolvent mixtures with the solute dissolved on it, as shown below [13-15].

Algebraic manipulation of the basic expressions presented by Newman [16] leads to expressions for the Kirkwood-Buff integrals (in $\mathrm{cm}^{3} \mathrm{~mol}^{-1}$ ) for the individual solvent components in terms of some thermodynamic quantities as shown in equations (3) and (4) [12, 15, 16]:

$$
\begin{aligned}
& G_{\mathrm{PG}, \mathrm{A}}=R T \kappa_{T}-V_{\mathrm{A}}+x_{\mathrm{W}} V_{\mathrm{W}} D / Q \\
& G_{\mathrm{W}, \mathrm{A}}=R T \kappa_{T}-V_{\mathrm{A}}+x_{\mathrm{PG}} V_{\mathrm{PG}} D / Q
\end{aligned}
$$

Where $\kappa_{T}$ is the isothermal compressibility of the PG + water solvent mixtures (in $\mathrm{GPa}^{-1}$ ), $V_{\mathrm{PG}}$ and $V_{W}$ are the partial molar volumes of the solvents in the mixtures (in $\mathrm{cm}^{3} \mathrm{~mol}^{-1}$ ), similarly, $V_{\mathrm{A}}$ is the partial molar volume of solute in these mixtures (in $\mathrm{cm}^{3} \mathrm{~mol}^{-1}$ ). The function $D$ is the derivative of the standard molar Gibbs energies of transfer of the drug (from neat water to $P G$ + water mixtures) with respect to the proportion of $P G$ in the mixtures (in $\mathrm{kJ} \mathrm{mol}^{-1}$, as also is $R T$ ). Otherwise, the function $Q$ involves the second derivative of the excess molar Gibbs energy of mixing of the two solvents $\left(G_{\mathrm{PG}+\mathrm{W}}^{E x c}\right)$ with respect to the water proportion in the mixtures (also in $\mathrm{kJ} \mathrm{mol}^{-1}$ ) [14, 15]:

$$
\begin{aligned}
& D=\left(\frac{\partial \Delta_{\mathrm{tr}} G_{(\mathrm{A}, \mathrm{W} \rightarrow \mathrm{PG}+\mathrm{W})}^{0}}{\partial x_{\mathrm{PG}}}\right)_{T, p} \\
& Q=R T+x_{\mathrm{PG}} x_{\mathrm{W}}\left(\frac{\partial^{2} G_{\mathrm{PG}, \mathrm{W}}^{E x c}}{\partial x_{\mathrm{W}}^{2}}\right)_{T, p}
\end{aligned}
$$

Because the dependence of $\kappa_{T}$ on mixtures composition is not known for a lot of the systems investigated normally in pharmacy, and because of the small contribution of $R T \kappa_{T}$ to the $\mid \mathrm{KBI}$ method, the dependence of $\kappa_{T}$ on composition could be approximated by considering additive behavior, according to $\kappa_{T, \text { mix }}=\sum_{i=1}^{n} x_{i} \kappa_{T, i}^{0}$, where $x_{i}$ is the mole fraction of component $i$ in the mixture and $\kappa_{T, i}^{0}$ is the isothermal compressibility of the pure component $i$. For $P G$ + water mixtures the preferential solvation parameter can be calculated from the Kirkwood-Buff integrals as follows $[10,17]$ : 


$$
\delta x_{\mathrm{PG}, \mathrm{A}}=\frac{x_{\mathrm{PG}} x_{\mathrm{W}}\left(G_{\mathrm{PG}, \mathrm{A}}-G_{\mathrm{W}, \mathrm{A}}\right)}{x_{\mathrm{PG}} G_{\mathrm{PG}, \mathrm{A}}+x_{\mathrm{W}} G_{\mathrm{W}, \mathrm{A}}+V_{\text {cor }}}
$$

Here, the correlation volume, $V_{\text {cor }}$, is obtained by means of the following expression [16, 17]:

$$
V_{\text {cor }}=2522.5\left(r_{\mathrm{A}}+0.1363\left(x_{\mathrm{PG}, \mathrm{A}}^{L} V_{\mathrm{PG}}+x_{\mathrm{W}, \mathrm{A}}^{L} V_{\mathrm{W}}\right)^{1 / 3}-0.085\right)^{3}
$$

Where $r_{\mathrm{A}}$ is the radius of the solute (in $\mathrm{nm}$ ). However, the definitive correlation volume requires iteration, because it depends on the local mole fractions. This iteration is done by replacing $\delta x_{\mathrm{PG}, \mathrm{A}}$ in the equation (2) to calculate $x_{\mathrm{PG}, \mathrm{A}}^{L}$ until a non-variant value of $V_{\text {cor }}$ is obtained [17].

For the QLQC method, the local mole fraction of PG around the ACP molecules is defined as [15]:

$$
\begin{aligned}
& x_{\mathrm{A}}^{L}=1 /\left[1+\left(N_{\mathrm{PGPG}} / N_{\mathrm{WW}}\right)^{0.5} \exp \left(\Delta E_{\mathrm{PGW}, \mathrm{A}} / 2 R T\right)\right] \\
& N_{\mathrm{PGPG}} / N_{\mathrm{WW}}=\left[x_{\mathrm{PG}}-N_{\mathrm{PGW}} / Z\left(N_{\mathrm{PG}}+N_{\mathrm{W}}\right)\right] / \\
& {\left[x_{\mathrm{W}}-N_{\mathrm{PGW}} / Z\left(N_{\mathrm{PG}}+N_{\mathrm{W}}\right)\right]} \\
& \frac{N_{\mathrm{PGW}}}{Z\left(N_{\mathrm{PG}}+N_{\mathrm{W}}\right)}=\frac{1-\left[1-4 x_{\mathrm{PG}} x_{\mathrm{W}}\left(1-\exp \left\{-\Delta E_{\mathrm{PGW}} / R T\right\}\right)\right]^{0.5}}{2\left[1-\exp \left(-\Delta E_{\mathrm{PGW}} / R T\right)\right]} \\
& \Delta E_{\mathrm{PGW}, \mathrm{A}}=\Delta_{\mathrm{tr}} G_{(\mathrm{A}, \mathrm{W} \rightarrow P G)}^{\mathrm{o}} / Z
\end{aligned}
$$

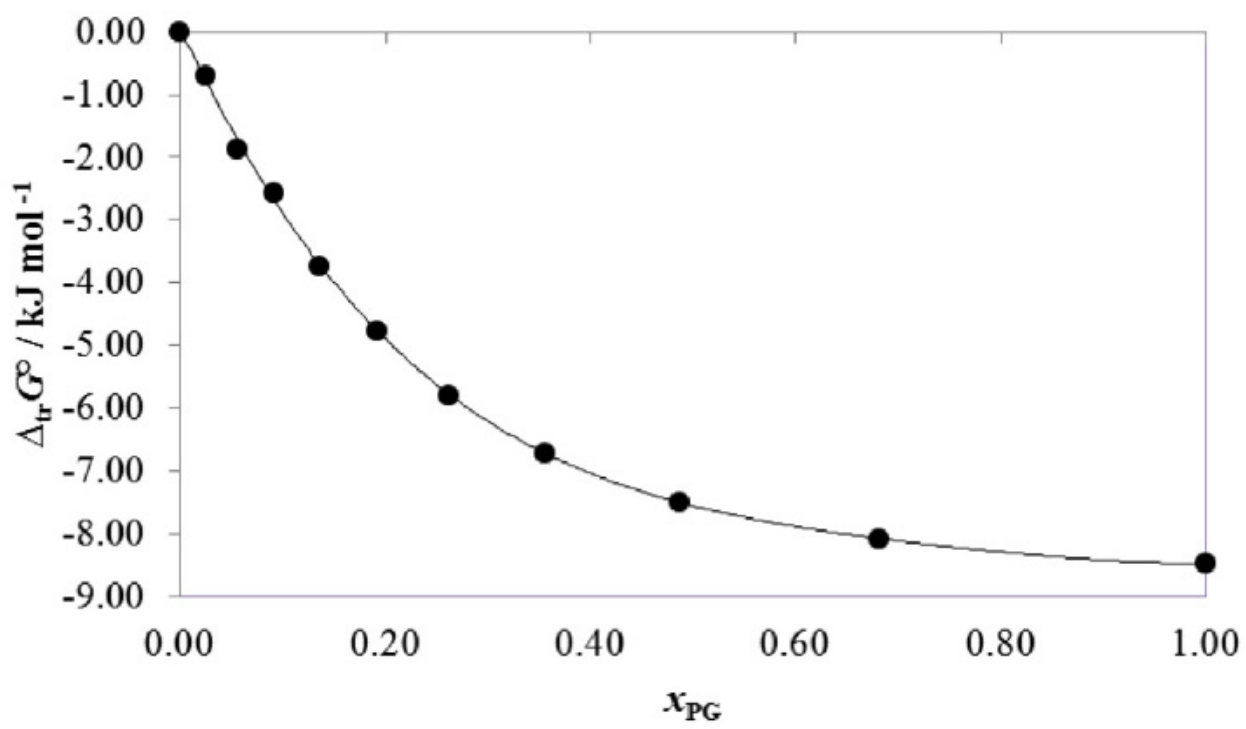

Figure 2: Gibbs energy of transfer of acetaminophen from neat water to propylene glycol + water co-solvent mixtures at 303.15 K. $\exp \left(\Delta E_{\mathrm{PGW}} / R T\right)=\left[\left(2 \exp \left\{-G_{\mathrm{PGW}(x=0.5)}^{E x c} / Z R T\right\}\right)-1\right]^{2}$ assumed as 10. $N_{\mathrm{PG}}$ and $N_{\mathrm{W}}$ are the number of molecules of both components in the bulk, whereas, $N_{\text {PGPG }}, N_{\text {WW }}$, and $N_{\text {PGW }}$ are the number of neighboring pairs of these molecules in the quasi lattice. Equation (11) expresses the difference in the molar neighbor interaction energies of ACP with the propylene glycol and water, $\Delta E_{\mathrm{PGW}, \mathrm{A}}$, by the molar Gibbs energy of transfer from water to propylene glycol per neighboring lattice. $\Delta E_{\mathrm{PGW}}$ denotes the molar energy of interaction of solvent on neighboring quasi-lattice sites. It is important to keep in mind that just the Gibbs energy of the drug transfer between the neat solvents and the excess Gibbs energy of mixing at equimolar composition of both solvents are required for this method.

\section{RESULTS AND DISCUSSION}

The solubility of ACP in $P G$ + water mixtures was taken from Jiménez and Martínez [2]. Standard molar Gibbs energy of transfer of this drug from neat water to $P G+$ water mixtures is calculated and correlated to regular quartic polynomials from the drug solubility data by using equation (9). This degree of polynomials was chosen based on some significant statistical parameters, such as, determination coefficients and residual analyses (values not shown here). All calculations were made by using MS Excel ${ }^{\circledR}$ and TableCurve 2D v5.01. Otherwise, Figure 2 shows the
In these equations, the lattice parameter $Z$ is usually 
Table 1: Gibbs Energy of Transfer $\left(\mathrm{kJ} \mathrm{mol}^{-1}\right)^{\mathrm{a}}$ of Acetaminophen from Neat Water to Propylene Glycol + Water CoSolvent Mixtures at Several Temperatures

\begin{tabular}{|c|c|c|c|}
\hline$X_{P G}{ }^{b}$ & $293.15 K$ & $303.15 K$ & $313.15 K$ \\
\hline 0.0000 & 0.00 & 0.00 & 0.00 \\
\hline 0.0256 & -0.51 & -0.70 & -0.52 \\
\hline 0.0559 & -1.54 & -1.86 & -1.68 \\
\hline 0.0921 & -2.37 & -2.58 & -2.36 \\
\hline 0.1364 & -3.63 & -3.75 & -3.59 \\
\hline 0.1915 & -4.79 & -4.77 & -4.66 \\
\hline 0.2621 & -5.78 & -5.80 & -5.58 \\
\hline 0.3559 & -6.75 & -6.71 & -6.46 \\
\hline 0.4865 & -7.53 & -7.49 & -7.25 \\
\hline 0.6807 & -8.12 & -8.08 & -7.85 \\
\hline 1.0000 & -8.42 & -8.47 & -8.26 \\
\hline
\end{tabular}

${ }^{a}$ Solubility values used in the calculation were taken from Ref. [2].

${ }^{b} x_{P G}$ is the mole fraction of propylene glycol in the propylene glycol + water co-solvent mixtures free of acetaminophen

Table 2: Coefficients of the Equation (9) $\left(\mathrm{kJ} \mathrm{mol}^{-1}\right)$ Applied to Gibbs Energy of Transfer of Acetaminophen from Neat Water to Propylene Glycol + Water Co-Solvent Mixtures at Several Temperatures

\begin{tabular}{|c|c|c|c|}
\hline Coefficient & $\mathbf{2 9 3 . 1 5} \mathbf{K}$ & $\mathbf{3 0 3 . 1 5} \mathrm{K}$ & $\mathbf{3 1 3 . 1 5} \mathbf{K}$ \\
\hline \hline$a$ & 0.18 & 0.05 & -33.97 \\
\hline$b$ & -33.84 & -34.99 & 57.92 \\
\hline$c$ & 51.06 & 60.97 & -47.05 \\
\hline$d$ & -33.12 & -51.10 & 14.70 \\
\hline
\end{tabular}

Gibbs energy of transfer behavior at $303.15 \mathrm{~K}$ whereas Table 1 shows the behavior at all the temperatures studied. The coefficients of the polynomials are shown in Table 2.

$\Delta_{\mathrm{tr}} G_{\mathrm{A}, \mathrm{W} \rightarrow \mathrm{PG}+\mathrm{W}}^{0}=R T \ln \left(\frac{x_{\mathrm{A}, \mathrm{W}}}{x_{\mathrm{A}, \mathrm{PG}+\mathrm{W}}}\right)=$

$a+b x_{\mathrm{PG}}+c x_{\mathrm{PG}}^{2}+d x_{\mathrm{PG}}^{3}+e x_{\mathrm{PG}}^{4}$

Thus $D$ values are calculated from the first derivative of polynomial models (Equation 10) solved according to the co-solvent mixtures composition. This procedure was done varying by 0.05 in mole fraction of PG. $D$ values are reported in Table 3.

$D=b+2 c x_{\mathrm{PG}}+3 d x_{\mathrm{PG}}^{2}+4 e x_{\mathrm{PG}}^{3}$

The physicochemical properties of the $P G+$ water binary mixtures, i.e. $Q$ and $R T \kappa_{T}$ values, as well as the partial molar volumes of $P G$ and water, at the three temperatures considered here, were taken from the literature [17].

Partial molar volumes of non-electrolyte drugs are not frequently reported in the literature. This is due to the big uncertainty in its determination because of the low solubilities exhibited, in particular in aqueous media. For this reason, in a first approach the molar volume of ACP is considered here as independent of co-solvent composition and temperature, as it is calculated according to the groups contribution method proposed by Fedors [18]. Thus, this value has been considered as reported by Ahumada et al. as $V_{\mathrm{A}}=$ $111.2 \mathrm{~cm}^{3} \mathrm{~mol}^{-1}$ [19] and used to estimate the preferential solvation of this drug in ethanol + water mixtures [6]. Otherwise, the radius of the drug molecule was also taken from the literature as $r_{\mathrm{A}}=0.353 \mathrm{~nm}$ [6].

Table 4 shows that the $G_{P G, A}$ and $G_{W, A}$ values are negative at all temperatures under study. In water-rich 
Table 3: $D$ Values $\left(\mathrm{kJ} \mathrm{mol}^{-1}\right)$ of Acetaminophen in Propylene Glycol + Water Co-Solvent Mixtures at Several Temperatures

\begin{tabular}{|c|c|c|c|}
\hline$X_{P G}$ & $293.15 \mathrm{~K}$ & $303.15 \mathrm{~K}$ & $313.15 \mathrm{~K}$ \\
\hline 0.00 & -33.84 & -34.99 & -33.97 \\
\hline 0.05 & -28.98 & -29.27 & -28.52 \\
\hline 0.10 & -24.59 & -24.27 & -23.74 \\
\hline 0.15 & -20.66 & -19.93 & -19.57 \\
\hline 0.20 & -17.16 & -16.21 & -15.98 \\
\hline 0.25 & -14.06 & -13.06 & -12.92 \\
\hline 0.30 & -11.36 & -10.42 & -10.34 \\
\hline 0.35 & -9.02 & -8.25 & -8.20 \\
\hline 0.40 & -7.02 & -6.50 & -6.46 \\
\hline 0.45 & -5.35 & -5.12 & -5.07 \\
\hline 0.50 & -3.97 & -4.05 & -3.99 \\
\hline 0.55 & -2.87 & -3.25 & -3.18 \\
\hline 0.60 & -2.03 & -2.68 & -2.58 \\
\hline 0.65 & -1.42 & -2.27 & -2.16 \\
\hline 0.70 & -1.03 & -1.98 & -1.88 \\
\hline 0.75 & -0.82 & -1.76 & -1.68 \\
\hline 0.80 & -0.78 & -1.55 & -1.53 \\
\hline 0.85 & -0.89 & -1.32 & -1.37 \\
\hline 0.90 & -1.13 & -1.01 & -1.18 \\
\hline 0.95 & -1.46 & -0.56 & -0.89 \\
\hline 1.00 & -1.88 & 0.06 & -0.48 \\
\hline
\end{tabular}

Table 4: $G_{\mathrm{PG}, \mathrm{A}}$ and $G_{\mathrm{W}, \mathrm{A}}$ Values $\left(\mathrm{cm}^{3} \mathrm{~mol}^{-1}\right)$ for Acetaminophen in Propylene Glycol + Water Co-Solvent Mixtures at Several Temperatures

\begin{tabular}{|c|c|c|c|c|c|c|}
\hline$X_{P G}$ & \multicolumn{3}{|c|}{$G_{\mathrm{PG}, \mathrm{A}}$} & \multicolumn{3}{|c|}{$G_{\mathrm{W}, \mathrm{A}}$} \\
\hline 0.00 & -360.9 & -361.5 & -347.1 & -110.1 & -110.0 & -110.0 \\
\hline 0.10 & -269.9 & -272.2 & -273.5 & -179.6 & -181.1 & -182.1 \\
\hline 0.15 & -235.7 & -235.8 & -238.6 & -198.0 & -198.6 & -201.1 \\
\hline 0.30 & -165.6 & -161.9 & -162.7 & -208.0 & -201.9 & -203.6 \\
\hline 0.35 & -150.6 & -147.3 & -147.5 & -201.1 & -193.9 & -194.6 \\
\hline 0.40 & -138.9 & -136.5 & -136.3 & -191.2 & -184.6 & -184.2 \\
\hline 0.45 & -130.0 & -128.7 & -128.3 & -179.5 & -175.2 & -173.9 \\
\hline 0.50 & -123.3 & -123.1 & -122.7 & -167.2 & -166.6 & -164.7 \\
\hline 0.70 & -112.0 & -113.6 & -113.3 & -130.4 & -147.6 & -144.7 \\
\hline 0.75 & -111.3 & -112.7 & -112.5 & -127.4 & -145.8 & -143.4 \\
\hline 0.80 & -111.0 & -111.9 & -111.8 & -127.7 & -144.0 & -142.6 \\
\hline 0.85 & -110.9 & -111.2 & -111.2 & -131.5 & -141.0 & -141.6 \\
\hline 0.90 & -110.8 & -110.6 & -110.7 & -139.1 & -135.4 & -139.2 \\
\hline 0.95 & -110.5 & -110.2 & -110.2 & -150.6 & -125.3 & -133.8 \\
\hline 1.00 & -110.0 & -110.0 & -109.9 & -166.6 & -108.2 & -123.5 \\
\hline
\end{tabular}


Table 5: Correlation Volume for Acetaminophen in Propylene Glycol + Water Co-Solvent Mixtures at Several Temperatures

\begin{tabular}{|c|c|c|c|}
\hline \multirow{2}{*}{$X_{P G}$} & \multicolumn{3}{|c|}{$V_{\text {cor }} / \mathrm{cm}^{3} \mathrm{~mol}^{-1}$} \\
\hline & $293.15 \mathrm{~K}$ & $303.15 \mathrm{~K}$ & $313.15 \mathrm{~K}$ \\
\hline 0.00 & 619 & 619 & 621 \\
\hline 0.05 & 653 & 654 & 656 \\
\hline 0.10 & 701 & 703 & 705 \\
\hline 0.15 & 755 & 758 & 761 \\
\hline 0.25 & 863 & 866 & 870 \\
\hline 0.30 & 913 & 916 & 920 \\
\hline 0.35 & 960 & 963 & 967 \\
\hline 0.40 & 1004 & 1008 & 1012 \\
\hline 0.60 & 1170 & 1177 & 1182 \\
\hline 0.65 & 1210 & 1218 & 1224 \\
\hline 0.70 & 1251 & 1259 & 1265 \\
\hline 0.75 & 1292 & 1300 & 1306 \\
\hline 0.80 & 1332 & 1340 & 1347 \\
\hline 0.85 & 1373 & 1380 & 1387 \\
\hline 0.90 & 1413 & 1419 & 1426 \\
\hline 0.95 & 1452 & 1458 & 1466 \\
\hline
\end{tabular}

mixtures $G_{P G, A}$ values are bigger in magnitude in comparison with $G_{W, A}$ values but in mixtures with similar solvent proportions and in PG-rich mixtures these values are lower than $G_{W, A}$.

In order to use the $\mathrm{IKBI}$ method, the correlation volume was iterated three times by using the equations (2), (7) and (8) to obtain the values reported in Table 5. It is interesting to note that this value is almost independent on temperature in water-rich mixtures but increases in some extent in PG-rich mixtures as expectable according to the respective molar expansibilities [20].

According to Figure 3 , the values of $\delta x_{P G, A}$ vary nonlinearly with the $P G$ concentration in the aqueous mixtures at $303.15 \mathrm{~K}$ (Filled dots). Addition of PG to water tends to make negative the $\delta x_{\mathrm{PG}, \mathrm{A}}$ values of $\mathrm{ACP}$ from the pure water up to the mixture 0.20 in mole fraction of $P G$ reaching a minimum in $x_{1}=0.10\left(\delta x_{P G, A}\right.$ $\left.=-1.60 \times 10^{-2}\right)$. In the case of ethanol + water mixtures the minimum value was also obtained in the mixture with $x_{\mathrm{EtOH}}=0.10$ but in that case the solvation parameter was bigger in magnitude $\left(\delta x_{\mathrm{EtOH}}=-3.24 \mathrm{x}\right.$ $10^{-2}$ ) [6]. As was indicated previously, possibly the structuring of water molecules around the non-polar groups of this drug, i.e. the hydrophobic hydration of the aromatic ring and methyl groups, contributes to lowering of the net $\delta x_{P G, A}$ to negative values in these water-rich mixtures (Table 5).

In the mixtures with composition $0.20<x_{P G}<1.00$, the local mole fractions of $P G$ are greater than those for water. In this way, the co-solvent action may be related to the breaking of the ordered structure of water (aggregates stabilized by hydrogen bonding) around the non-polar moieties of the drug, which could increases the solvation of the ACP and exhibiting a maximum value near to $x_{P G}=0.40$, i.e. $\delta x_{P G, A}=1.37 x$ $10^{-2}$ at $303.15 \mathrm{~K}$.

As has been indicated earlier, ACP could act in solution as a Lewis acid due to the hydrogen atoms in its $-\mathrm{OH}$ and $-\mathrm{NH}$ groups (Figure 1 ) in order to establish 


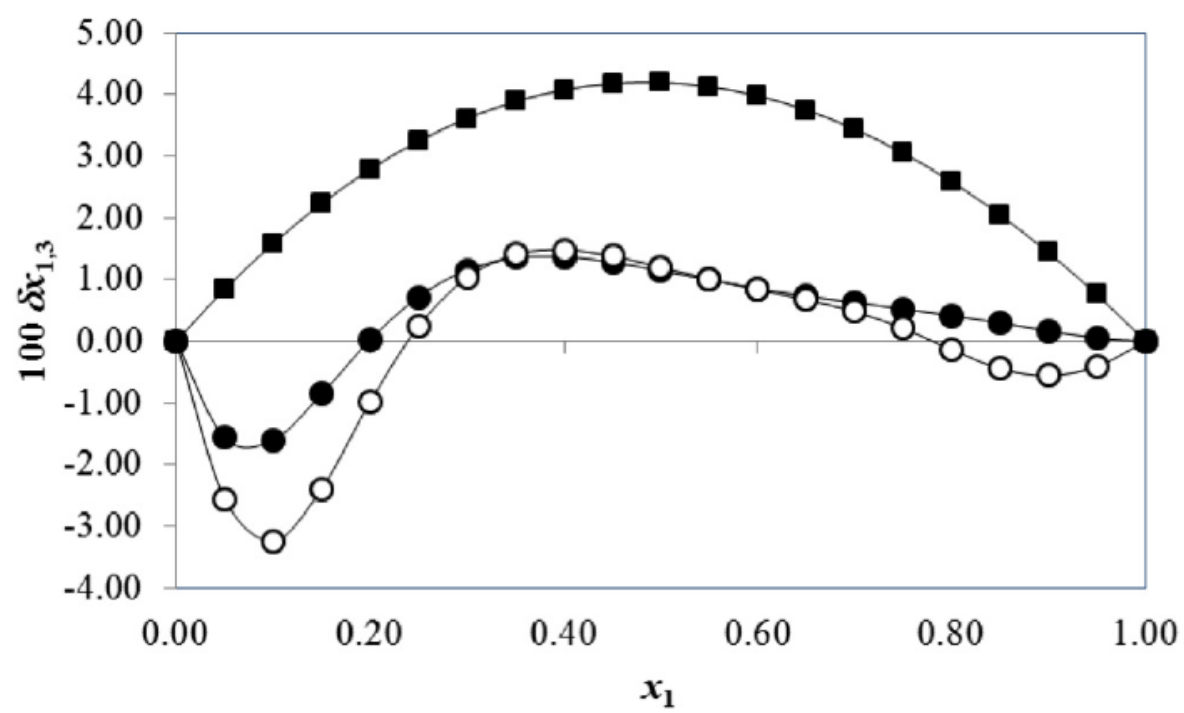

Figure 3: $\delta x_{1,3}$ values for acetaminophen in propylene glycol + water $(\bullet: \mathrm{IKBI} ; \mathbf{m}: \mathrm{QLQC})$ and ethanol + water $(\circ$ : IKBI) cosolvent mixtures at $303.15 \mathrm{~K}$. Here 1 stands for co-solvent (propylene glycol or ethanol), 2 for water, and 3 for acetaminophen.

hydrogen bonds with proton-acceptor functional groups in the solvents (oxygen atoms in $-\mathrm{OH}$ ). In addition, this drug could act as a Lewis base due to free electron pairs in oxygen atoms of hydroxyl and carbonyl groups (Figure 1) to interact with acidic hydrogen atoms present in both solvents. In this context, ACP has two hydrogen-bonding donor and two hydrogen-bonding acceptor groups [6].

According to the preferential solvation results, it is conjecturable that in intermediate composition and in PG-rich mixtures, the ACP is acting as Lewis acid with PG molecules because this co-solvent is more basic than water, i.e. the Kamlet-Taft hydrogen bond acceptor parameters are $\beta=0.78$ for $P G$ and 0.47 for water [21]. On the other hand, it is interesting to compare these results with those reported for ACP in ethanol-rich mixtures (Empty dots in Figure 3), where the drug is preferentially solvated by water $\left(0.78<x_{\mathrm{EtOH}}\right.$ $<1.00)$ [6]. In that case this drug could be acting mainly as a Lewis base in front to water because the KamletTaft hydrogen bond donor parameters are, $\alpha=1.17$ for water and 0.86 for ethanol, respectively [6, 22]. Thus, water is more acidic than ethanol. Nevertheless, the reasons for this difference between $P G+$ water and ethanol + water mixtures remain unclear.

On the other hand, in order to use the QLQC method, the excess Gibbs energy of mixing values of the equimolar mixture of both solvents were used as follows, $-7.03 \times 10^{-2},-4.81 \times 10^{-2}$, and $-1.35 \times 10^{-2} \mathrm{~kJ}$ $\mathrm{mol}^{-1}$, at the same temperatures [10]. According to the QLQC method (Table 6 and Figure 3), ACP is preferentially solvated by the co-solvent in all the mixtures. Clearly the QLQC $\delta x_{P G, A}$ values are bigger than those obtained by using the IKBI method in all the mixtures. Maximum is found in the mixture with $W_{P G}=$ 0.50 with $\delta x_{P G, A}=4.192 \times 10^{-2}$ at $303.15 \mathrm{~K}$. Therefore, as has been indicated in the literature, the IKBI method is more adequate than QLQC method to discriminate the effect of the co-solvent composition on the local mole fraction of the solvents around the drug molecules, in particular in the water-rich mixtures $[9$, 10]. Nevertheless, it is important to keep in mind that QLQC requires only two specific experimental values, i.e. the Gibbs energy of transfer of ACP from water to co-solvent and the excess Gibbs energy of mixing in the co-solvent mixture with composition $x_{1}=0.50$, and therefore, it is more easy to use.

\section{CONCLUSION}

In this work some explicit expressions for estimating the local mole fraction of propylene glycol and water around acetaminophen molecules were derived based on the IKBI and QLQC methods applied to the reported equilibrium solubility values of this drug in some $P G+$ water mixtures. Thereby, according to the IKBI method this drug is preferentially solvated by water in waterrich mixtures but preferentially solvated by $P G$ in mixtures with intermediate composition and in PG-rich mixtures at all temperatures considered. These results are in agreement with those described previously for this drug, which were based in more classical thermodynamic treatments, i.e. quantities for drug solution, mixing and overall solvation processes [2]. Otherwise, according to the QLQC method, this drug would be preferentially solvated by the co-solvent in all 
Table 6: IKBI and QLQC Values for Acetaminophen in Propylene Glycol + Water Co-Solvent Mixtures at Several Temperatures

\begin{tabular}{|c|c|c|c|c|c|c|}
\hline \multirow{2}{*}{$X_{P G}$} & \multicolumn{3}{|c|}{ IKBI, $100 \delta x_{\mathrm{PG}, \mathrm{A}}$} & \multicolumn{3}{|c|}{ QLQC, $100 \delta x_{P G, A}$} \\
\hline & $293.15 \mathrm{~K}$ & $303.15 \mathrm{~K}$ & $313.15 \mathrm{~K}$ & $293.15 \mathrm{~K}$ & $303.15 \mathrm{~K}$ & $313.15 \mathrm{~K}$ \\
\hline 0.00 & 0.000 & 0.000 & 0.000 & 0.000 & 0.000 & 0.000 \\
\hline 0.05 & -1.540 & -1.563 & -1.523 & 0.858 & 0.843 & 0.805 \\
\hline 0.10 & -1.587 & -1.600 & -1.601 & 1.618 & 1.586 & 1.513 \\
\hline 0.15 & -0.873 & -0.857 & -0.865 & 2.280 & 2.233 & 2.127 \\
\hline 0.20 & 0.009 & 0.023 & 0.036 & 2.846 & 2.785 & 2.648 \\
\hline 0.25 & 0.750 & 0.723 & 0.749 & 3.317 & 3.243 & 3.080 \\
\hline 0.30 & 1.242 & 1.156 & 1.179 & 3.695 & 3.609 & 3.424 \\
\hline 0.35 & 1.479 & 1.350 & 1.358 & 3.982 & 3.886 & 3.682 \\
\hline 0.40 & 1.504 & 1.370 & 1.358 & 4.179 & 4.074 & 3.855 \\
\hline 0.45 & 1.378 & 1.284 & 1.253 & 4.286 & 4.176 & 3.947 \\
\hline 0.50 & 1.164 & 1.146 & 1.101 & 4.307 & 4.192 & 3.958 \\
\hline 0.55 & 0.917 & 0.996 & 0.942 & 4.241 & 4.125 & 3.890 \\
\hline 0.60 & 0.679 & 0.856 & 0.797 & 4.091 & 3.976 & 3.745 \\
\hline 0.65 & 0.482 & 0.735 & 0.676 & 3.858 & 3.746 & 3.525 \\
\hline 0.70 & 0.341 & 0.628 & 0.576 & 3.542 & 3.437 & 3.231 \\
\hline 0.75 & 0.256 & 0.527 & 0.488 & 3.146 & 3.050 & 2.864 \\
\hline 0.80 & 0.220 & 0.420 & 0.402 & 2.671 & 2.587 & 2.427 \\
\hline 0.85 & 0.209 & 0.300 & 0.305 & 2.117 & 2.050 & 1.921 \\
\hline 0.90 & 0.196 & 0.171 & 0.195 & 1.487 & 1.438 & 1.346 \\
\hline 0.95 & 0.142 & 0.053 & 0.083 & 0.780 & 0.755 & 0.706 \\
\hline 1.00 & 0.000 & 0.000 & 0.000 & 0.000 & 0.000 & 0.000 \\
\hline
\end{tabular}

the possible mixtures. Nevertheless, it is important to consider that the IKBI method is more rigorous than QLQC and more reliable results are thus obtained with the former method.

\section{REFERENCES}

[1] Raffa RB. Analgesic, antipyretic, and anti-inflammatory drugs. In: Gennaro A, editor. Remington: The Science and Practice of Pharmacy. 21st ed. Philadelphia: Lippincott Williams \& Wilkins 2005.

[2] Jiménez J, Martinez F. Thermodynamic study of the solubility of acetaminophen in propylene glycol + water cosolvent mixtures. J Braz Chem Soc 2006; 17: 125-134. http://dx.doi.org/10.1590/S0103-50532006000100018

[3] Jouyban A. Handbook of Solubility Data for Pharmaceuticals. Boca Raton, FL: CRC Press; 2010.

[4] Rubino JT. Cosolvents and cosolvency. In: Swarbrick J, Boylan JC, editors. Encyclopedia of Pharmaceutical Technology, vol 3. New York: Marcel Dekker, Inc.; 1988.

[5] Jiménez J, Martínez F. Thermodynamic magnitudes of mixing and solvation of acetaminophen in ethanol + water cosolvent mixtures. Rev Acad Colomb Cienc 2006; 30: 8799.
[6]

Delgado DR, Peña MA, Martínez F. Preferential solvation of acetaminophen in ethanol + water solvent mixtures according to the inverse Kirkwood-Buff integrals method. Rev Colomb Cienc Quim Farm 2013; 42: 298-314.

[7] Marcus Y. Preferential solvation of ibuprofen and naproxen in aqueous 1,2-propanediol. Acta Chim Slov 2009; 56: 40-44.

[8] Ruidiaz MA, Delgado DR, Martínez F, Marcus Y. Solubility and preferential solvation of indomethacin in 1,4-dioxane + water solvent mixtures. Fluid Phase Equilib 2010; 299: 259265.

http://dx.doi.org/10.1016/j.fluid.2010.09.027

[9] Delgado DR, Holguín AR, Almanza OA, Martínez F, Marcus $Y$. Solubility and preferential solvation of meloxicam in ethanol + water mixtures. Fluid Phase Equilib. 2011; 305: 8895.

http://dx.doi.org/10.1016/j.fluid.2011.03.012

[10] Holguín AR, Delgado DR, Martínez F, Marcus Y. Solution thermodynamics and preferential solvation of meloxicam in propylene glycol + water mixtures. J. Solution Chem 2011; 40: 1987-1999.

http://dx.doi.org/10.1007/s10953-011-9769-0

[11] Ben-Naim A. Theory of preferential solvation of nonelectrolytes. Cell Biophysics 1988; 12: 255-269. http://dx.doi.org/10.1007/BF02918361

[12] Ben-Naim A. Preferential solvation in two- and in threecomponent systems. Pure \& Appl Chem 1990; 62: 25-34. http://dx.doi.org/10.1351/pac199062010025 
[13] Marcus Y. Solubility and solvation in mixed solvent systems. Pure \& Appl Chem 1990; 62: 2069-2076. http://dx.doi.org/10.1351/pac199062112069

[14] Marcus Y. Solvent Mixtures: Properties and Selective Solvation. New York: Marcel Dekker, Inc.; 2002.

[15] Marcus $Y$. On the preferential solvation of drugs and PAHs in binary solvent mixtures. J Mol Liq 2008; 140: 61-67. http://dx.doi.org/10.1016/j.molliq.2008.01.005

[16] Newman KE. Kirkwood-Buff solution theory: Derivation and applications. Chem Soc Rev 1994; 23: 31-40. http://dx.doi.org/10.1039/cs9942300031

[17] Delgado DR, Peña MA, Martínez F. Preferential solvation of some sulfonamides in propylene glycol + water solvent mixtures according to the IKBI and QLQC methods. J Solution Chem 2014; 43: 360-374. http://dx.doi.org/10.1007/s10953-014-0130-2

[18] Fedors RF. A method for estimating both the solubility parameters and molar volumes of liquids. Polym Eng Sci 1974; 14: 147-154.

http://dx.doi.org/10.1002/pen.760140211
[19] Ahumada EA, Delgado DR, Martínez F. Acetaminophen solubility in polyethylene glycol $400+$ water mixtures according to the extended Hildebrand solubility approach. Rev Colomb Quím 2012; 41: 433-438.

[20] Jiménez J, Martínez F. Study of some volumetric properties of 1,2-propanediol + water mixtures at several temperatures. Rev Colomb Cienc Quím Farm 2005; 34: 46-57.

[21] Kamlet MJ, Taft RW. The solvatochromic comparison method. I. The beta-scale of solvent hydrogen-bond acceptor (HBA) basicities. J Am Chem Soc 1976; 98: 377-383. http://dx.doi.org/10.1021/ja00418a009

[22] Taft RW, Kamlet MJ. The solvatochromic comparison method. II. The alpha-scale of solvent hydrogen-bond donor (HBD) acidities. J Am Chem Soc 1976; 98: 2886-2894. http://dx.doi.org/10.1021/ja00426a036 